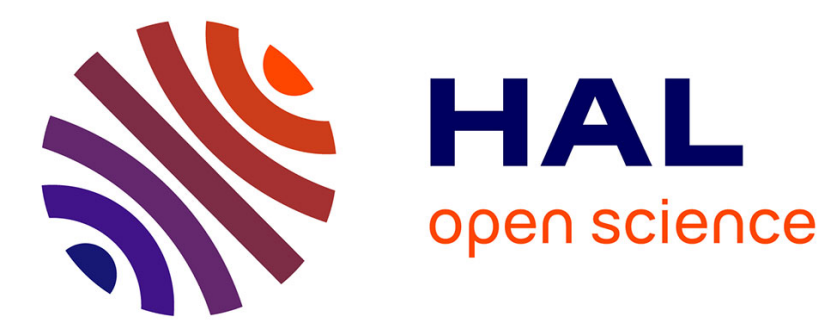

\title{
Mieux communiquer avec les patients non hospitalisés
}

Didier Sciard, Marc Beaussier

\section{To cite this version:}

Didier Sciard, Marc Beaussier. Mieux communiquer avec les patients non hospitalisés. Praticien en Anesthésie Réanimation, 2016, 20 (2), pp.73-77. 10.1016/j.pratan.2016.02.008 . hal-01298645

\section{HAL Id: hal-01298645 https: / hal.sorbonne-universite.fr/hal-01298645}

Submitted on 6 Apr 2016

HAL is a multi-disciplinary open access archive for the deposit and dissemination of scientific research documents, whether they are published or not. The documents may come from teaching and research institutions in France or abroad, or from public or private research centers.
L'archive ouverte pluridisciplinaire HAL, est destinée au dépôt et à la diffusion de documents scientifiques de niveau recherche, publiés ou non, émanant des établissements d'enseignement et de recherche français ou étrangers, des laboratoires publics ou privés. 


\section{Mieux communiquer avec les patients non hospitalisés}

D Sciard, M Beaussier

Département d'Anesthésie-Réanimation chirurgicale. Unité de Chirurgie Ambulatoire.

Hôpital St-Antoine. Groupe Hospitalier Est-Parisien. APHP.

Sorbonne Universités. UPMC Univ Paris 06. France.

Tel : $+33(0) 149282362$

Adresse électronique : didier.sciard@aphp.fr

La chirurgie ambulatoire est basée sur un concept d'organisation centrée sur le patient (1). Elle s'inscrit dans une continuité entre la ville et l'unité de chirurgie ambulatoire. Un plan de communication entre tous les acteurs de cette prise en charge doit être mis en place que ce soit avant, pendant ou après l'acte opératoire. Le groupe de travail HAS/ANAP a défini le parcours patient en 6 étapes (2) (3). A chacune de ces étapes il faudra avoir une communication claire avec le patient, son accompagnant ainsi qu'avec tous les acteurs de soins qu'ils soient médicaux ou paramédicaux, en ville ou à l'hôpital. En tant qu'acteur de ses soins le patient devra être informé de toutes les étapes de son séjour pour qu'il participe à sa prise en charge péri-opératoire.

Les 6 étapes de cette prise en charge sont :

- Eligibilité du patient

- Organisation de la prise en charge du patient éligible

- Actions à mener la veille ou l'avant-veille de l'intervention prévue (J-1 ou J-2)

- Prise en charge du patient le jour de l'intervention (JO)

- Suivi immédiat après la sortie $(\mathrm{J}+1)$

- Suivi du patient à distance de la sortie.

A chacune de ces étapes il faudra connaitre, anticiper et maitriser les risques auxquels le patient sera exposé.

\section{1) Eligibilité du patient}

Pour que le patient soit éligible à une prise en charge ambulatoire il faut qu'il réponde à des critères médicaux et chirurgicaux d'une part, psycho-sociaux et environnementaux d'autre part. Qui mieux que le médecin traitant peut apprécier avec la famille du patient certains critères d'éligibilité. Pour cela il faut que le chirurgien correspondant du médecin traitant informe celui-ci avec précision du geste qui sera effectué et de sa prise en charge en ambulatoire.

\section{2) Organisation de la prise en charge du patient éligible}


La programmation par le chirurgien est une étape importante qui doit tenir compte des impératifs de ce mode de prise en charge. Il faut passer du « je vous opère le... » à " quand puis-je vous opérer ? " car c'est à ce moment que le patient ayant été informé des conséquences de cette chirurgie sur son autonomie devra s'enquérir de la disponibilité de son accompagnant et des éventuels soins à domicile et de la nature et la durée du handicap lié à la chirurgie. C'est aussi à ce moment qu'il faut anticiper quelques consignes de sortie telles que la nécessité de béquilles ou la prise en charge de la douleur.

Lors de cette consultation il faudra dans certain cas communiquer largement avec l'accompagnant lorsqu'il s'agit de la prise en charge d'un enfant, d'une personne âgée et/ou dépendante ou lorsqu'il existe une barrière linguistique.

Une fiche de liaison avec l'anesthésiste permettra aussi de préciser la nature et la durée prévisible de l'acte ainsi que certains besoins spécifiques comme ceux liés à la position opératoire souhaitée.

La consultation d'anesthésie suit celle du chirurgien et prendra en compte tout ce qui a été décidé lors de cette consultation. Le patient et son accompagnant éventuel se verront réitérer toutes les informations concernant la prise en charge, la nécessité d'avoir un accompagnant pour la sortie et possiblement pour la première nuit.

Lui seront aussi données les informations spécifiques sur le mode d'anesthésie choisi avec en particulier la prise en compte du risque de nausées/vomissements, de rétention urinaire ou de douleur postopératoire. Si une technique d'analgésie par un bloc périnerveux continu est envisagée, toutes les informations concernant le suivi à domicile de cette infusion lui seront données.

Le patient doit sortir de la consultation du chirurgien et de l'anesthésiste avec en sa possession toutes les informations et documents nécessaires à sa prise en charge. Ces documents seront regroupés dans un dossier spécifique encore appelé passeport ambulatoire.

Idéalement à l'issu de ces consultations, le patient prendra contact avec l'unité de chirurgie ambulatoire pour visualiser le parcours qu'il devra effectuer le jour de l'intervention et recevoir les consignes du personnel de l'UCA l'informant entre autre de la procédure d'appel le ou les jours précédents l'intervention.

\section{3) Actions à mener la veille ou l'avant-veille de l'intervention prévue (J-1 ou J-2)}

Dans les jours précédents la chirurgie et habituellement la veille (appel J-1), le patient doit être recontacté par le secrétariat de l'UCA soit par téléphone soit par un système d'appel automatisé. Cet appel de la veille vise à réduire les dysfonctionnements de prise en charge.

Les principaux objectifs sont de :

- Dépister les modifications de l'état de santé du patient

- Informer de l'heure de rendez-vous du patient et de son accompagnant

- Rappeler les consignes préopératoires

- Diminuer le risque d'annulation 
- Préparer les patients à l'intervention

Cet appel actuellement réalisé dans la plupart des centres par téléphone présente plusieurs inconvénients. Il est chronophage et représente un "temps-coût" en secrétariat ou personnels soignants non négligeable pour la structure. Il est en général opérateur-dépendant, n'est pas toujours compatible avec la disponibilité du patient et dépend donc du moment de réception et ne résout pas le problème de la barrière linguistique si elle existe.

Le nombre croissant de patients pris en charge en CA incite à développer des techniques de rappel automatisées avec pour but de :

- Décharger les équipes infirmières de tâches répétitives à faible valeur ajoutée

- Identifier les patients présentant une anomalie dans le suivi

- Allouer les ressources aux seuls patients qui en ont besoin

Les nouvelles techniques relatives à l'informatique connectée à l'internet (NTIC) au service de la santé permettent de développer des nouveaux outils de suivi médical basés sur ces nouvelles technologies avec pour objectif une meilleure observance et une meilleure efficience des protocoles de prise en charge.

Plusieurs études ont montré que l'utilisation d'un système de communication par un service de message court " Short Message Service » (SMS) permet de réduire significativement les annulations et d'augmenter le respect des consignes préopératoires (4). Deng et al ont ainsi montré une diminution significative du taux d'annulation dans le groupe des patients recevant un SMS avec $40 \%$ de probabilité en moins d'annulation. De même le respect des consignes était plus important dans le groupe SMS comparé au groupe contrôle (5).

En pédiatrie, l'utilisation d'un programme de préparation des enfants et de leurs parents via un système d'information par internet a montré son efficacité pour réduire l'anxiété préopératoire des enfants (6) (7).

Une étude récente du "Credoc » souligne que la "m-health" ou " médecine connectée » va bouleverser les pratiques (8). Le graphique $n^{\circ} 1$ montre que l'utilisation des NTIC varie en fonction de l'âge mais ne chute vraiment qu'après 70 ans. Les Smartphones ou l'utilisation courante d'internet sont encore inégalement répartis dans la population mais seront sans doute le moyen de communication privilégié dans le futur car la majorité des moins de 30 ans sont « connectés » (graphique $\mathrm{n}^{\circ} 2$ ).

En attendant, le SMS peut être le moyen privilégié pour une automatisation. En effet cette étude du Credoc montre que $89 \%$ de la population française possède un téléphone portable et que $78 \%$ sont utilisateurs habituels des SMS.

Les avantages des SMS par rapport à l'appel téléphonique classique sont :

- $\quad 97 \%$ sont lus dans les 5 secondes

- Choix du moment et du nombre de lectures 
- Choix dans la langue de lecture

- Trace numérique du contact

Dans le service de chirurgie ambulatoire de St Antoine nous avons mis en place avec la société Calmedica et son système Memoquest ${ }^{\circledR}$ (outil de dialogue automatisé entre les professionnels de santé et le patient) un dialogue automatisé par SMS entre notre centre de soins et le patient de chirurgie ambulatoire. Nous avons défini un protocole de suivi en indiquant les questions que l'on veut poser au patient ambulatoire, les dates et les heures d'envoi ainsi que les réponses attendues. Toute réponse non attendue, ou toute non-réponse déclenchera une alerte chez le patient et le professionnel de santé.

Le dialogue automatisé consistera donc à suivre un algorithme défini par le médecin.

Le système Memoquest ${ }^{\circledR}$ va :

Envoyer des SMS au patient aux dates et heures convenues

$>$ Recueillir les réponses

Activer les questions suivantes ou les alertes en fonction des réponses

Avant de mettre en place ce système d'appel J-1 automatisé nous avons mené une étude visant à comparer le rappel des consignes préopératoires par SMS avec l'appel individuel réalisé par un soignant, sur une hypothèse de " non-infériorité ". Par rapport à l'appel téléphonique, l'utilisation de SMS à J-1 en chirurgie ambulatoire améliore le respect des consignes préopératoires (CPO) et cette modalité de rappel des CPO a satisfait la majorité des patients. (Tableau 1)

\section{4) Prise en charge du patient après l'intervention (J0)}

Les instructions sont dispensées au patient après la phase de récupération post anesthésique de façon à s'assurer que ces consignes sont bien comprises. Cette communication se fera lors de la visite de sortie par le chirurgien et l'anesthésiste. Cette communication orale des consignes de sortie sera renforcée par une communication d'un certain nombre de documents mis à la disposition du patient dans son passeport ambulatoire. Le respect de l'article D 6124-304 du code de la santé publique doit être absolue. Ces dispositions prévoient que le patient soit en possession d'un bulletin de sortie mentionnant notamment des recommandations sur les conduites à tenir en matière de surveillance post-opératoire et les coordonnées de l'établissement de santé assurant la permanence et la continuité des soins.

Figureront donc dans son passeport ambulatoire une :

Une communication avec un éventuel médecin (CRH / CRO)

Une communication à l'aide d'une fiche de liaison pour des soins infirmiers

U Une communication des documents relatifs à l'éducation du patient 
Une communication des informations relatives à la continuité des soins et à l'accessibilité de structures adéquates en cas de besoin (permanence téléphonique dans un service hospitalier de référence)

\section{5) Suivi immédiat après la sortie $(\mathrm{J}+1)$}

La continuité des soins après chirurgie implique un suivi du patient à domicile notamment en ce qui concerne les premières $24 \mathrm{~h}$ postopératoires (9). Ce suivi est généralement assuré par un appel téléphonique à domicile et vise à s'enquérir du respect des consignes de sortie, de l'efficacité des traitements de sortie, de l'absence de complications liées à la chirurgie et à l'anesthésie (saignement, douleurs, nausées/vomissements...) et du degré de satisfaction du patient quant à sa prise en charge.

Cet appel est en principe fait par une infirmière. Comme pour l'appel J-1 le temps qui y est consacré est très important et ne fait l'objet d'une réponse par le patient que dans environ $50 \%$ des cas. Lorsque le volume de patients traités quotidiennement devient important, il est quasi impossible d'être exhaustif. Il devient alors nécessaire d'être sélectif sur le type de patient et/ou de pathologie qui nécessite un appel téléphonique, et de développer un système d'appel automatisé à J+1.

Dans cet esprit nous avons mis en place à St Antoine, avec la même société, un système d'appel automatisé de certains patients à $\mathrm{J}+1$. Pour l'instant nous avons limité cet appel à certaines chirurgies de la main (canal carpien, kystes, doigt à ressaut, Dupuytren non compliqué, plaies simples, AMO) et du pied, à la stomatologie et à certaines chirurgies générales (lipome, examen anal) (Annexe 1). Cet appel automatisé à J+1 est en cours d'évaluation.

Cet appel du lendemain automatisé permet aussi de récupérer le maximum de questionnaires de satisfaction. Une étude australienne a montré qu'une enquête de satisfaction envoyée par SMS et internet avait un taux de réponse de $69 \%$, largement supérieur au taux de réponse obtenu par appel téléphonique (10).

Le suivi du patient en postopératoire peut parfois être étendu sur plusieurs jours notamment dans le cadre du suivi de cathéters périnerveux mais aussi possiblement pour un suivi de drains, redons, ou sonde urinaire à domicile.

Dans notre centre nous travaillons avec un prestataire extérieur pour le suivi à domicile par des infirmières libérales des patients bénéficiant de cathéters périnerveux. Le patient sort de l'unité avec un dossier de suivi de son cathéter, la société ayant pris contact avec lui dans les jours précédents l'intervention.

Le patient à domicile peut aussi bénéficier pour son suivi médical ou chirurgical de la télémédecine. La télémédecine est réglementée en France depuis 2009 (article L.6316-1 du Code de la santé publique). Dans le cadre de la chirurgie ambulatoire cette approche médicale via les nouvelles technologies peut permettre d'effectuer une surveillance de l'état du patient comme par exemple un suivi du site chirurgical, d'un bloc nerveux continu ou de paramètres vitaux comme le suivi du pouls et de la température (fuite anastomotique) (11) (12).

Des systèmes d'auto-surveillance à domicile après intervention via un appareil mobile (smartphone ou tablette) et sur internet existent. Plusieurs sociétés proposent une solution informatique de suivi 
des patients à domicile avant ou après chirurgie ou médecine ambulatoire. Parmi celles-ci il y a les systèmes AMBUCARE ${ }^{\circledR}$ ou SOVINTY ${ }^{\circledR}$ implémentés par des collègues libéraux.

\section{Conclusion}

Communiquer avec le patient à toutes les étapes de sa prise en charge en chirurgie ambulatoire est donc d'une très grande importance pour minimiser les risques associés à ce mode de prise en charge. Lors de la journée ambulatoire de l'AFCA en 2011, Christian Saout, Président du Collectif Inter associatif Sur la Santé (CISS), nous a donné le point de vue des usagers sur la chirurgie ambulatoire en insistant sur la nécessité d'une information du patient plus claire et plus exhaustive.

\section{Bibliographie}

1. IAAS. Chirurgie ambulatoire. Recommandations sur le suivi d'indicateurs cliniques de qualité. http://www.iaas-med.com/index.php/iaas-recommendations/clinical-indicators.

2. HAS. Ensemble pour le développement de la Chirurgie Ambulatoire. Recommandations organisationnelles http://www.has-sante.fr/portail/upload/docs/application/pdf/201306/recommandations vd.pdf 2013.

3. Beaussier M, Marchand-Maillet F, Dufeu N, Sciard D. Organizational aspects to optimize patient's ambulatory pathway. Curr Opin Anaesthesiol 2015;28:636-41.

4. Pratap JN, Varughese AM, Mercurio P, Lynch T, Lonnemann T, Ellis A, Rugg J, Stone WR, Bedinghaus $C$. Reducing Cancelations on the Day of Scheduled Surgery at a Children's Hospital. Pediatrics. 2015;135(5):1292-9

5. Kain ZN, Fortier MA, Chorney JM, Mayes L. Web-based tailored intervention for preparation of parents and children for outpatient surgery (WebTIPS): development. Anesth Analg. 2015; 120(4):905-14.

6. Fortier MA, Bunzli E, Walthall J, Olshansky E, Saadat H, Santistevan R, Mayes L, Kain ZN. Web-based tailored intervention for preparation of parents and children for outpatient surgery (WebTIPS): formative evaluation and randomized controlled trial. Anesth Analg. $2015 ; 120(4): 915-22$

7. Belavy D. A mobile telephone-based SMS and internet survey system for self-assessment in Australian anaesthesia: experience of a single practitioner. Anaesth Intensive Care. 2014;42(6):771-6

8. Deng $X$, Wang Y, Zhu T, Zhang W, Yin Y, Ye L. Short message service (SMS) can enhance compliance and reduce cancellations in a sedation gastrointestinal endoscopy center : a prospective randomized controlled trial. J Med Syst. 2015;39(1):169.

9. La diffusion des technologies de L'information et de La communication dans La société française (2013). www.credoc.fr N² 297 novembre 2013 
10. Beaussier M, Vons C. [Post-hospital home care after ambulatory surgery]. Presse Med 2013;43:305-8

11. Rebibo $L^{1}$, Blot $C^{1}$, Verhaeghe $P^{1}$, Cosse $C^{1}$, Dhahri $A^{1}$, Regimbeau JM ${ }^{2}$. Effect of perioperative management on short-term outcomes after sleeve gastrectomy: a 600-patient single-center cohort study. Surg Obes Relat Dis. 2014;10(5):853-8.

12. Rebibo $\mathrm{L}^{1}$, Dhahri $\mathrm{A}^{1}$, Badaoui $\mathrm{R}^{2}$, Dupont $\mathrm{H}^{2}$, Regimbeau $\mathrm{JM}^{3}$. Laparoscopic sleeve gastrectomy as day-case surgery (without overnight hospitalization). Surg Obes Relat Dis. 2015;11(2):335-42. 
Tableau 1

\begin{tabular}{|c|c|c|c|}
\hline & Groupe "Appel" & Groupe "SMS" & $p$ \\
\hline & $n(\%)$ & $n(\%)$ & \\
\hline Critière de jugement principal : Absence de tout dysfonctionnement dans la prise en charge à I0 & $184(61,1)$ & $222(74,5)$ & $<0,001$ \\
\hline Présence d'au moins un dysfonctionnement ci-dessous dans la prise en chargè à J0 & $117(38,9)$ & $76(25,5)$ & $\langle 0,001$ \\
\hline Annulation J0 & $12(4,0)$ & $12(4,0)$ & \\
\hline Absence d'accompagnant & $0(0,0)$ & $0(0,0)$ & \\
\hline Retard $>30$ minutes & $31(10,3)$ & $3(1,0)$ & \\
\hline Patient non à jeun & $46(15,3)$ & $35(11,7)$ & \\
\hline Douche non prise & $0(0,0)$ & $1(0,3)$ & \\
\hline Ongles non courts & $36(12,0)$ & $20(6,7)$ & \\
\hline Non retraits des bijoux/Vernis & $14(4,7)$ & $11(3,7)$ & \\
\hline
\end{tabular}


Graphique 1

Utilisation des NTIC en fonction de l'âge (Source Credoc Juin 2012)

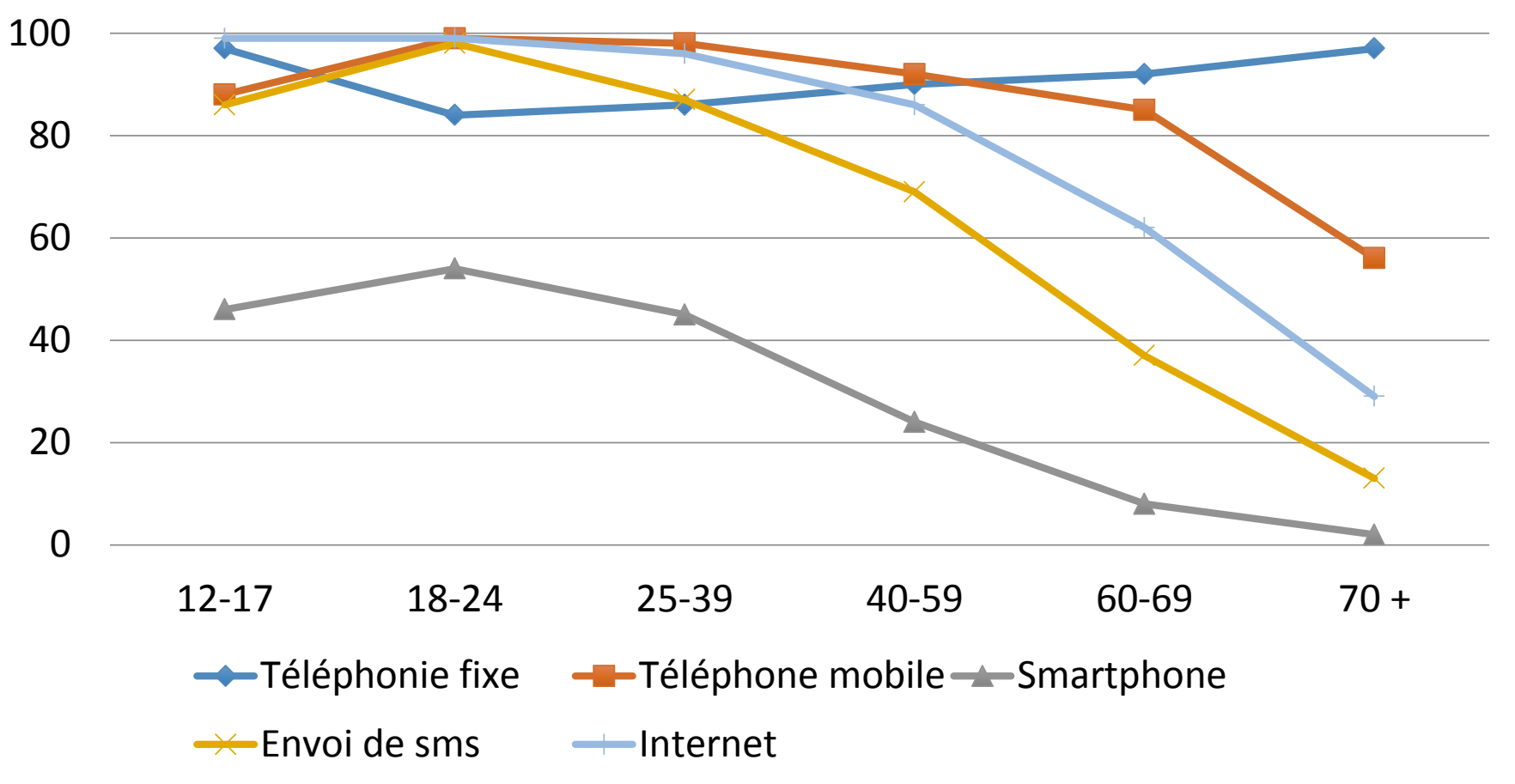




\section{Global Media End of 2014}

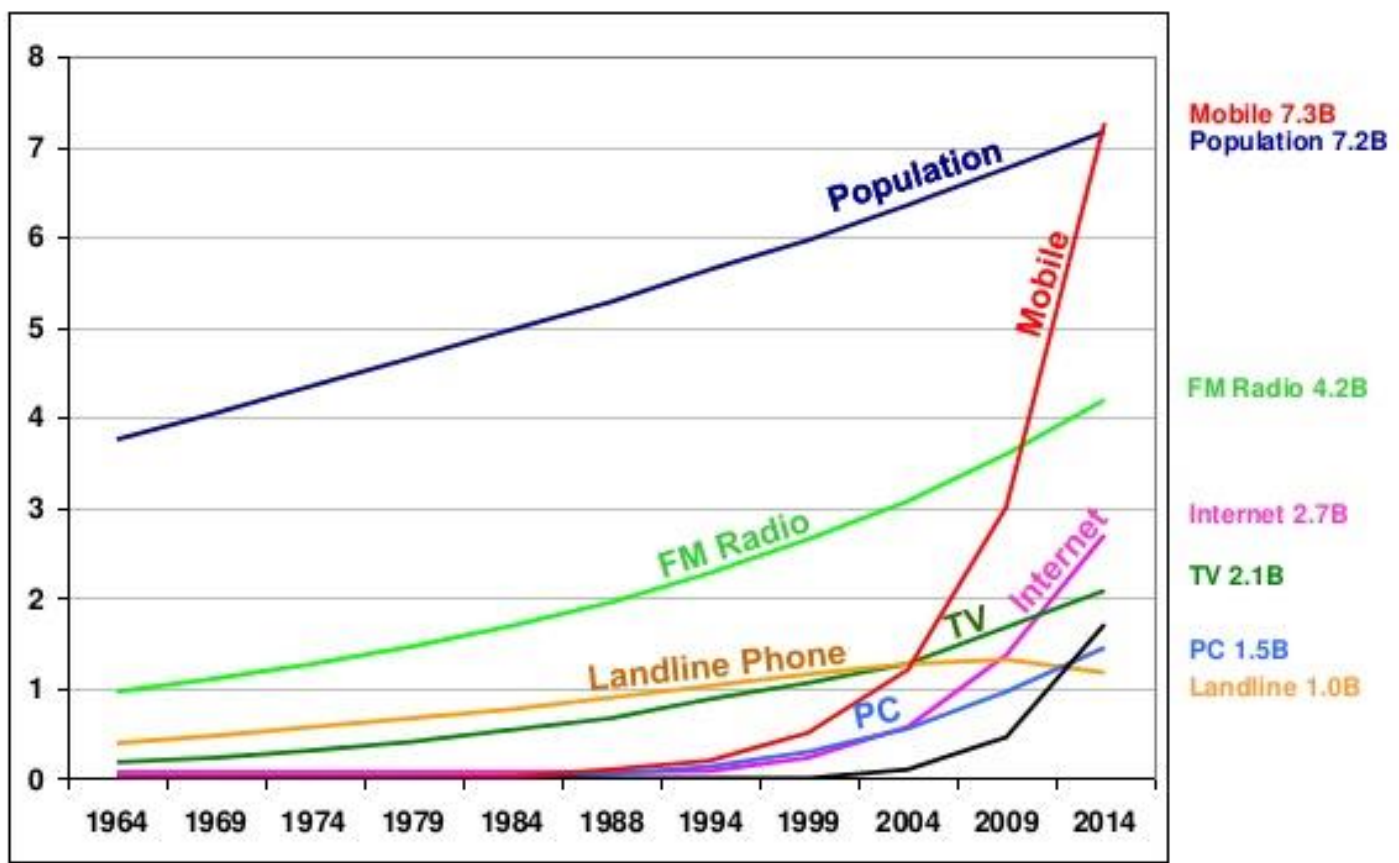

Source: TomiAhonen Almanac 2014

Copyright @ Tomi T Ahonen 2015 
Annexe 1

- Automatisation de l'appel J+1 en chirurgie ambulatoire

\section{Information des patients bénéficiant de l'automatisation par SMS de l'appel du lendemain de l'intervention}

Après chirurgie ambulatoire, il est recommandé de prévoir un contact le lendemain de l'intervention afin de s'assurer du bon déroulement des suites opératoires. Si vous en êtes d'accord, nous vous proposons de réaliser ce contact par SMS automatisé.

Voici les messages que vous allez recevoir. Nous vous demandons de les lire attentivement et d'y répondre tel qu'indiqué dans le message.

1ère question : Avez-vous actuellement une douleur non calmée par les médicaments ? Répondez simplement un chiffre de 0 à 10 ( 0 pas de douleur du tout... 10 douleur insupportable)

2ème question : Avez-vous eu des nausées-vomissements, des saignements ou des vertiges importants, un trouble du sommeil anormal ? Répondez simplement Oui si vous avez eu l'un de ces symptômes

\section{3ème question :}

- Si vous avez été opéré de la main : Si vos doigts sont gonflés ou que vous avez l'impression que le pansement est trop serré : Gardez la main levée, bougez les doigts, desserrez un peu le pansement. Si les symptômes persistent dans $48 \mathrm{~h}$ ou pour tout autre problème ou question appelez-nous au 01 71970174 du lundi au vendredi de $8 \mathrm{~h}$ à $17 \mathrm{~h}$

- Pour les autres interventions : Pour tout problème ou question appelez-nous au 0171970174 du lundi au vendredi de $8 \mathrm{~h}$ à $17 \mathrm{~h}$

4eme question : Avez-vous été satisfait de votre prise en charge dans l'unité de chirurgie ambulatoire ? Répondez simplement un chiffre de 0 à 10 ( 0 pas du tout satisfait... 10 très satisfait) 


\section{Protocole J+1 Hôpital Saint-Antoine}

\section{Main}

\begin{tabular}{|l|l|l|}
\hline Heure & Question & Conditions d'alerte \\
\hline $10 \mathrm{~h}$ & $\begin{array}{l}\text { Avez-vous actuellement une douleur non calmée par les } \\
\text { médicaments ? Répondez simplement un chiffre de 0 à 10 } \\
\text { (0 pas de douleur du tout... 10 douleur insupportable) }\end{array}$ & $>3$ \\
\hline $10 \mathrm{~h}$ & $\begin{array}{l}\text { Avez-vous eu des nausées-vomissements, des saignements } \\
\text { ou des vertiges importants, un trouble du sommeil } \\
\text { anormal? Répondez simplement Oui si vous avez eu l'un de } \\
\text { ces symptômes }\end{array}$ & Oui \\
\hline $10 \mathrm{~h}$ & $\begin{array}{l}\text { Si vos doigts sont gonflés ou que vous avez l'impression que } \\
\text { le pansement est trop serré : Gardez la main levée, bougez } \\
\text { les doigts, desserrez un peu le pansement. Si les symptômes } \\
\text { persistent dans 48h ou pour tout autre problème ou } \\
\text { question appelez-nous au 01 71 97 01 74 du lundi au } \\
\text { vendredi de 8h à 17h }\end{array}$ & $\begin{array}{l}\text { Aun } \\
\text { 14hense à }\end{array}$ \\
\hline $14 \mathrm{~h}$ & $\begin{array}{l}\text { Avez-vous été satisfait de votre prise en charge dans l'unité } \\
\text { de chirurgie ambulatoire ? Répondez simplement un chiffre } \\
\text { de 0 à 10 (0 pas du tout satisfait... 10 très satisfait) }\end{array}$ & \\
\hline
\end{tabular}

\section{Général}

\begin{tabular}{|l|l|l|}
\hline Heure & Question & Alerte \\
\hline $10 \mathrm{~h}$ & $\begin{array}{l}\text { Avez-vous actuellement une douleur non calmée par les } \\
\text { médicaments ? Répondez simplement un chiffre de 0 à 10 } \\
\text { (0 pas de douleur du tout... 10 douleur insupportable) }\end{array}$ & $>3$ \\
\hline $10 \mathrm{~h}$ & $\begin{array}{l}\text { Avez-vous eu des nausées-vomissements, des saignements } \\
\text { ou des vertiges importants, un trouble du sommeil } \\
\text { anormal? Répondez simplement Oui si vous avez eu l'un de } \\
\text { ces symptômes }\end{array}$ & Oui \\
\hline $10 \mathrm{~h}$ & $\begin{array}{l}\text { Pour tout problème ou question appelez-nous au 01 71 97 } \\
\text { 01 74 du lundi au vendredi de 8h à 17h }\end{array}$ & $\begin{array}{l}\text { Aucune réponse à } \\
14 \mathrm{~h}\end{array}$ \\
\hline $14 \mathrm{~h}$ & $\begin{array}{l}\text { Avez-vous été satisfait de votre prise en charge dans l'unité } \\
\text { de chirurgie ambulatoire ? Répondez simplement un chiffre } \\
\text { de 0 à 10 (0 pas du tout satisfait... 10 très satisfait) }\end{array}$ & \\
\hline
\end{tabular}

